\title{
On submultiplicative constants of an algebra
}

\author{
James S. Cook ${ }^{1 *}$ and Khang V. Nguyen ${ }^{1}$
}

${ }^{*}$ Correspondence: jcook4@liberty.edu

'Liberty University, Lynchburg, United States

\begin{abstract}
If $\mathcal{A}$ is a finite-dimensional commutative associative real algebra with norm $\|\cdot\|$ then we say that the rth submultiplicative constant of $\mathcal{A}$ is the smallest constant $m_{r}(\mathcal{A})$ for which $\left\|x_{1} x_{2} \cdots x_{r}\right\| \leq m_{r}(\mathcal{A})\left\|x_{1}\right\|\left\|x_{2}\right\| \cdots\left\|x_{r}\right\|$. For a product algebra, we show that there exist zero divisors where equality is attained in the inequality defining $m_{r}(\mathcal{A})$. We also study $\rho_{\mathcal{A}}=\lim \sup _{r \rightarrow \infty} \sqrt[r]{m_{r}(\mathcal{A})}$. We explain how $\rho_{\mathcal{A}}$ appears in the generalization of the Cauchy-Hadamard criterion for hypercomplex power series. We find the submultiplicative constants and $\rho_{\mathcal{A}}$ for the real group algebra of the cyclic group of order $n$ as well as the complicated numbers $\mathcal{C}_{n}=\left\{a_{1}+a_{2} k+\cdots+a_{n} k^{n-1} \mid a_{i} \in \mathbb{R}, k^{n}=-1\right\}$ with Euclidean norm. Submultiplicative constants for the $n$-dual numbers $\Delta_{n}$ with Euclidean norm are also calculated or conjectured for $n \leq 6$. We show, for $n \geq 2, \rho_{\Delta_{n}}=1$ for $\Delta_{n}$ given the $p$-norm.
\end{abstract}

Keywords: Cyclic group algebra; Complicated numbers; Hypercomplex analysis; Submultiplicative norms

\section{Introduction}

Suppose $V_{1}, \ldots, V_{r}$ are finite-dimensional spaces over either $\mathbb{R}$ or $\mathbb{C}$, and $X$ is a normed space. It is known that any bounded multilinear map $T: V_{1} \times V_{2} \times \cdots \times V_{r} \rightarrow X$ is continuous. Furthermore, finite-dimensional $V_{1}, \ldots, V_{r}$ have compact unit ball whose Cartesian products are likewise compact. Therefore, the extreme value theorem yields that the norm $\|T\|_{\text {op }}=\sup \left\{\left\|T\left(v_{1}, v_{2}, \ldots, v_{r}\right)\right\|:\left\|v_{i}\right\| \leq 1,1 \leq i \leq r\right\}$ is attained by evaluation of $T$ at some point in the Cartesian product of the unit-balls of $V_{1}, \ldots, V_{r}$. Since $r$-fold multiplication on an algebra $\mathcal{A}$ provides an $\mathcal{A}$-valued $r$-linear map over $\mathcal{A}$, we have the following result:

Theorem 1.1 If $\mathcal{A}$ is a finite-dimensional unital associative algebra (with multiplication denoted as $\star)$ over $\mathbb{R}$ or $\mathbb{C}$ with a norm $\|\cdot\|$ then there exists a smallest constant $m_{r}(\mathcal{A}) \in \mathbb{R}$ for each $r \in \mathbb{N}, r \leq 2$ such that $\left\|x_{1} \star x_{2} \star \cdots \star x_{r}\right\| \leq m_{r}(\mathcal{A})\left\|x_{1}\right\|\left\|x_{2}\right\| \cdots\left\|x_{r}\right\|$ for all $x_{1}, x_{2}, \ldots, x_{r} \in \mathcal{A}$. Furthermore, there exist $y_{1}, \ldots, y_{r} \in \mathcal{A}$ for which equality is attained; $\left\|y_{1} \star y_{2} \star \cdots \star y_{r}\right\|=m_{r}(\mathcal{A})\left\|y_{1}\right\|\left\|y_{2}\right\| \cdots\left\|y_{r}\right\|$.

We call $m_{r}(\mathcal{A})$ the $r$ th submultiplicative constant of $\mathcal{A}$ (with respect to the norm $\|\cdot\|$ ). Usually, we denote $m_{2}(\mathcal{A})=m_{\mathcal{A}}$ and name it the submultiplicative constant. This improves the less sharp use of $m_{\mathcal{A}}$ in [1].

(c) The Author(s) 2019. This article is distributed under the terms of the Creative Commons Attribution 4.0 International License (http://creativecommons.org/licenses/by/4.0/), which permits unrestricted use, distribution, and reproduction in any medium, provided you give appropriate credit to the original author(s) and the source, provide a link to the Creative Commons license, and indicate if changes were made. 
Proposition 1.2 If $m_{\mathcal{A}}$ is the submultiplicative constant for $\mathcal{A}$ then $m_{r}(\mathcal{A}) \leq m_{\mathcal{A}}^{r-1}$. Also, if there exists $x \in \mathcal{A}$ for which $\left\|x^{r}\right\|=m_{\mathcal{A}}^{r-1}\|x\|^{r}$ for each $r \in \mathbb{N}$ then $m_{r}(\mathcal{A})=m_{\mathcal{A}}^{r-1}$.

Proof If $\|x \star y\| \leq m_{\mathcal{A}}\|x\|\|y\|$ for all $x, y \in \mathcal{A}$ then by repeated application of the inequality for the norm of the product of $x_{1}, x_{2}, \ldots, x_{r}$ we find

$$
\left\|x_{1} \star x_{2} \star \cdots \star x_{r}\right\| \leq m_{\mathcal{A}}^{r-1}\left\|x_{1}\right\|\left\|x_{2}\right\| \cdots\left\|x_{r}\right\| .
$$

Therefore, $m_{r}(\mathcal{A}) \leq m_{\mathcal{A}}^{r-1}$. Suppose there exists $x \in \mathcal{A}$ for which $\left\|x^{r}\right\|=m_{\mathcal{A}}^{r-1}\|x\|^{r}$. Setting $x_{1}=x_{2}=\cdots=x_{r}=x$ shows $(1)$ is sharp, hence $m_{r}(\mathcal{A})=m_{\mathcal{A}}^{r-1}$.

From these constants, we denote another constant related to the normed algebra $\mathcal{A}$

$$
\rho_{\mathcal{A}}=\limsup _{r \rightarrow \infty} \sqrt[r]{m_{r}(\mathcal{A})}
$$

Observe that $\rho_{\mathcal{A}}$ exists since $m_{r}(\mathcal{A}) \leq m_{\mathcal{A}}^{r-1}$, so $\rho_{\mathcal{A}} \leq m_{\mathcal{A}}$. In another direction, substitute $x_{1}=x_{2}=\cdots=x_{r}=1$ to obtain $\left\|1^{r}\right\| \leq m_{r}(\mathcal{A})\|1\|^{r}$, which implies $m_{r}(\mathcal{A}) \geq\|1\|^{-(r-1)}$ and

$$
\|1\|^{-1} \leq \rho_{\mathcal{A}} \leq m_{\mathcal{A}} .
$$

For a finite-dimensional associative real algebra $\mathcal{A}$, the radius of convergence for the geometric series $1+z+z^{2}+\cdots$ is shown to be at least $1 / m_{\mathcal{A}}$ in Theorem 4.5 of [2]. We offer an improved radius in what follows:

Theorem 1.3 If $\sum_{n=0}^{\infty} c_{n}\left(z-z_{0}\right)^{n}$ has $0<C=\lim \sup _{n \rightarrow \infty} \sqrt[n]{\left\|c_{n}\right\|}<\infty$ then the power series converges absolutely for $z \in \mathcal{A}$ such that $\left\|z-z_{0}\right\|<\left(C \rho_{A}\right)^{-1}$.

Proof For $C=\limsup _{n \rightarrow \infty} \sqrt[n]{\left\|c_{n}\right\|}$ and $\left\|z-z_{0}\right\|<\left(C \rho_{A}\right)^{-1}$ observe

$$
\limsup _{n \rightarrow \infty} \sqrt[n]{\left\|c_{n}\left(z-z_{0}\right)^{n}\right\|} \leq \limsup _{n \rightarrow \infty} \sqrt[n]{m_{n}(\mathcal{A})\left\|c_{n}\right\|\left\|z-z_{0}\right\|^{n}}=\rho_{A} C\left\|z-z_{0}\right\|<1
$$

Thus limsup $\sup _{n \rightarrow \infty} \sqrt[n]{\left\|c_{n}\left(z-z_{0}\right)^{n}\right\|}<1$, and we find $\sum_{n=0}^{\infty} c_{n}\left(z-z_{0}\right)^{n}$ converges absolutely by applying the Root Test for a series over an algebra (Theorem 4.2 of [2]).

The theorem above shows that convergence of a power series $\sum c_{n}\left(z-z_{o}\right)^{n}$ over an algebra $\mathcal{A}$ is governed both by the usual Cauchy-Hadamard criterion involving $\limsup _{n \rightarrow \infty} \sqrt[n]{\left\|c_{n}\right\|}$ and the constant $\rho_{\mathcal{A}}$. We note $\rho_{\mathcal{A}}$ depends on both $\mathcal{A}$ and the norm which $\mathcal{A}$ is given. In the remaining sections of this paper, we endeavor to calculate both $m_{r}(\mathcal{A})$ and $\rho_{\mathcal{A}}$ for several interesting algebras.

\section{Transfer principle}

Theorem 2.1 (Transfer Principle) If $\mathcal{A}, \mathcal{B}$ are finite-dimensional, unital, associative, normed algebras over $\mathbb{R}$ or $\mathbb{C}$ for which there exists an algebra isomorphism $\varphi: \mathcal{A} \rightarrow \mathcal{B}$ which is also a dilation (meaning there exists a constant $v>0$ so that $\|\varphi(x)\|=v\|x\|$ for all $x \in \mathcal{A})$. Then, for $r \geq 2, v^{r-1} \cdot m_{r}(\mathcal{B})=m_{r}(\mathcal{A})$ and $\rho_{\mathcal{A}}=v \cdot \rho_{\mathcal{B}}$. 
Proof Observe $\left\|\varphi\left(x_{1} \cdots x_{r}\right)\right\|=\left\|\varphi\left(x_{1}\right) \cdots \varphi\left(x_{r}\right)\right\| \leq m_{r}(\mathcal{B})\left\|\varphi\left(x_{1}\right)\right\| \cdots\left\|\varphi\left(x_{r}\right)\right\|$. Then as $\varphi$ is a dilation with constant $v$, it follows $\left\|x_{1} \cdots x_{r}\right\| \leq m_{r}(\mathcal{B}) v^{r-1}\left\|x_{1}\right\| \cdots\left\|x_{r}\right\|$. Recall $m_{r}(\mathcal{A})$ is the smallest constant for which $\left\|x_{1} \cdots x_{r}\right\| \leq m_{r}(\mathcal{A})\left\|x_{1}\right\| \cdots\left\|x_{r}\right\|$ for all $x_{1}, \ldots, x_{r} \in \mathcal{A}$. Therefore, $m_{r}(\mathcal{A}) \leq m_{r}(\mathcal{B}) v^{r-1}$. Observe $\varphi^{-1}: \mathcal{B} \rightarrow \mathcal{A}$ is a dilation with constant $\frac{1}{v}$ hence $m_{r}(\mathcal{B}) \leq m_{r}(\mathcal{A}) \frac{1}{v^{r-1}}$, from which we find $m_{r}(\mathcal{A}) \geq v^{r-1} m_{r}(\mathcal{B})$. Hence $v^{r-1} m_{r}(\mathcal{B})=m_{r}(\mathcal{A})$ and thus $\rho_{\mathcal{A}}=\limsup _{r \rightarrow \infty} \sqrt[r]{v^{r-1} m_{r}(\mathcal{B})}=v \lim \sup _{r \rightarrow \infty} \sqrt[r]{m_{r}(\mathcal{B})}=v \rho_{\mathcal{B}}$.

Theorem 2.2 (Product Algebra with Weighted $p$-norm) Let $\mathcal{A}_{1}, \ldots, \mathcal{A}_{s}$, be finitedimensional associative normed algebras with rth submultiplicative constant $m_{i, r}$ for $r \geq 2$ and with $\rho_{i}=\limsup _{r \rightarrow \infty} \sqrt[r]{m_{i, r}}$ for $1 \leq i \leq s$. If $\mathcal{A}=\mathcal{A}_{1} \times \mathcal{A}_{2} \times \cdots \times \mathcal{A}_{s}$ then define weighted p-norm with weights $a_{1}, \ldots, a_{s}>0$ by $\left\|\left(x_{1}, x_{2}, \ldots, x_{s}\right)\right\|=\max _{1 \leq i \leq s} a_{i}\left\|x_{i}\right\|$ for $p=\infty$ and

$$
\left\|\left(x_{1}, x_{2}, \ldots, x_{s}\right)\right\|=\left(\sum_{i=1}^{s} a_{i}^{p}\left\|x_{i}\right\|^{p}\right)^{\frac{1}{p}}
$$

for $p<\infty$. Then $\mathcal{A}$ with the weighted $p$-norm defines a normed associative algebra with $m_{r}(\mathcal{A})=\max _{1 \leq i \leq s}\left\{m_{i, r} / a_{i}^{r-1}\right\}$ and $\rho_{\mathcal{A}}=\max _{1 \leq i \leq s} \frac{\rho_{i}}{a_{i}}$.

Proof Given $x_{i, j} \in A_{i}$ for $1 \leq i \leq s, 1 \leq j \leq r$. Let $\mu_{r}=m_{r}(\mathcal{A})=\max _{1 \leq i \leq s}\left\{m_{i, r} / a_{i}^{r-1}\right\}$. Then

$$
\begin{aligned}
& \mu_{r}^{p} \prod_{j=1}^{r}\left\|\left(x_{1, j}, \ldots, x_{s, j}\right)\right\|^{p}=\mu_{r}^{p} \prod_{j=1}^{r} \sum_{i=1}^{s} a_{i}^{p}\left\|x_{i, j}\right\|^{p} \\
& \geq \mu_{r}^{p} \sum_{i=1}^{s} \prod_{j=1}^{r} a_{i}^{p}\left\|x_{i, j}\right\|^{p} \\
& =\mu_{r}^{p} \sum_{i=1}^{s}\left(a_{i}^{p}\right)^{r}\left(\prod_{j=1}^{r}\left\|x_{i, j}\right\|\right)^{p} \\
& \geq \max _{1 \leq i \leq s}\left\{\frac{m_{i, r}^{p}}{a_{i}^{(r-1) p}}\right\} \sum_{i=1}^{s}\left(a_{i}^{p}\right)^{r}\left(\frac{1}{m_{i, r}}\left\|\prod_{j=1}^{r} x_{i, j}\right\|\right)^{p} \\
& \geq \sum_{i=1}^{s} \frac{m_{i, r}^{p}}{a_{i}^{(r-1) p}} \cdot a_{i}^{r p} \cdot \frac{1}{m_{i, r}^{p}}\left\|\prod_{j=1}^{r} x_{i, j}\right\|^{p} \\
& =\sum_{i=1}^{s} a_{i}^{p}\left\|\prod_{j=1}^{r} x_{i, j}\right\|^{p} \\
& =\left\|\prod_{j=1}^{r}\left(x_{1, j}, \ldots, x_{s, j}\right)\right\|^{p} \text {. }
\end{aligned}
$$

From the above we derive

$$
\mu_{r} \prod_{j=1}^{r}\left\|\left(x_{1, j}, \ldots, x_{s, j}\right)\right\| \geq\left\|\prod_{j=1}^{r}\left(x_{1, j}, \ldots, x_{s, j}\right)\right\| .
$$

Let $k$ be an index between 1 and $s$ such that $\frac{m_{k, r}}{a_{k}^{r-1}}=\mu_{r}$ and, following the last sentence in Theorem 1.1, let $y_{k, j} \in \mathcal{A}_{k}(1 \leq j \leq r)$ be such that $m_{k, r} \prod_{j=1}^{r}\left\|y_{k, j}\right\|=\left\|\prod_{j=1}^{r} y_{k, j}\right\|$. Let $e_{k} \in \mathcal{A}$ 
denote $(0, \ldots, 1, \ldots, 0)$ where 1 is in the $k$ th entry. Then calculate

$$
\begin{aligned}
\mu_{r}^{p} \prod_{j=1}^{r}\left\|y_{k, j} e_{k}\right\|^{p} & =\left(a_{k} m_{k, r} \prod_{j=1}^{r}\left\|y_{k, j}\right\|\right)^{p} \\
& =\left(a_{k}\left\|\prod_{j=1}^{r} y_{k, j}\right\|\right)^{p} \\
& =\left\|\prod_{j=1}^{r}\left(y_{k, j} e_{k}\right)\right\|^{p},
\end{aligned}
$$

which indicates (6) is sharp, as equality is attained by setting $\left(x_{1, j}, \ldots, x_{s, j}\right)=y_{k, j} e_{k}$ for $j=$ $1, \ldots, r$. Thus $m_{r}(\mathcal{A})=\max _{1 \leq i \leq s}\left\{m_{i, r} / a_{i}^{r-1}\right\}$. Finally, observe

$$
\begin{aligned}
\rho_{\mathcal{A}} & =\limsup _{r \rightarrow \infty} \sqrt[r]{\max _{1 \leq i \leq s}\left\{m_{i, r} / a_{i}^{r-1}\right\}} \\
& =\limsup _{r \rightarrow \infty} \max _{1 \leq i \leq s}\left\{\frac{\sqrt[r]{m_{i, r}}}{\sqrt[r]{a_{i}^{r-1}}}\right\} \\
& =\max _{1 \leq i \leq s}\left\{\limsup _{r \rightarrow \infty} \frac{\sqrt[r]{m_{i, r}}}{\sqrt[r]{a_{i}^{r-1}}}\right\} \\
& =\max _{1 \leq i \leq s}\left\{\frac{\rho_{i}}{a_{i}}\right\} .
\end{aligned}
$$

Remark 2.3 If $\varphi: \mathcal{B} \rightarrow \mathcal{A}=\mathcal{A}_{1} \times \cdots \times \mathcal{A}_{s}$ is an isomorphism which is also a dilation then the proof given suggests a calculational method for finding an element $x \in \mathcal{B}$ for which $\left\|x^{2}\right\|=m_{\mathcal{B}}\|x\|^{2}$. In particular, select the element $y_{k, j} e_{k}$ for $r=2$ as in the proof and set $x=$ $\varphi^{-1}\left(y_{k, j} e_{k}\right)$. In this construction we will find that $x$ is a zero-divisor. However, if we consider algebras which are not product algebras then the situation is different. For example, in Theorem 5.1(1) we find that the unit $x=\sqrt{2}+\varepsilon$ gives $\|x\|=m_{\mathcal{A}}\|x\|^{2}$.

\section{Submultiplicative constants for cyclic group algebra}

The $n$-hyperbolic numbers are the algebra $\mathcal{H}_{n}=\mathbb{R}[x] /\left\langle x^{n}-1\right\rangle$ which is usually represented as

$$
\mathcal{H}_{n}=\left\{a_{0}+a_{1} j+\cdots+a_{n-1} j^{n-1} \mid a_{i} \in \mathbb{R}\right\}
$$

where we identify $j$ with $x+\left\langle x^{n}-1\right\rangle$ and 1 with $1+\left\langle x^{n}-1\right\rangle$. One may note that $\mathcal{H}_{n}$ is the real group algebra of the cyclic group of order $n$. In this section we derive the submultiplicative constants for hyperbolic numbers provided that $\mathcal{H}_{n}$ is given the norm

$$
\left\|a_{0}+a_{1} j+\cdots+a_{n-1} j^{n-1}\right\|=\sqrt{a_{0}^{2}+a_{1}^{2}+\cdots+a_{n-1}^{2}} .
$$




\section{1 $\mathcal{H}_{n}$ for even $\boldsymbol{n}$}

Notice that if $n=2 m$ then we have the following factorization of $x^{n}-1$ :

$$
x^{n}-1=(x-1)(x+1) \prod_{k=1}^{m-1}\left(x-e^{i \frac{k \pi}{m}}\right)\left(x-e^{-i \frac{k \pi}{m}}\right) \text {. }
$$

The Chinese remainder theorem and the factorization above suggests we form the isomorphism $\varphi: \mathcal{H}_{2 m} \rightarrow \mathbb{R}^{2} \times \mathbb{C}^{m-1}$ we describe next. If $p(j)=a_{0}+a_{1} j+\cdots+a_{n} j^{n-1} \in \mathcal{H}_{n}$ then $p(c)=a_{0}+a_{1} c+\cdots+a_{n} c^{n-1}$ for $c \in \mathbb{C}$, thus for $p(j) \in \mathcal{H}_{n}$ define

$$
\varphi(p(j))=\left(p(1), p(-1), p\left(e^{i \pi / m}\right), p\left(e^{2 i \pi / m}\right), \ldots, p\left(e^{(m-1) i \pi / m}\right)\right) .
$$

Observe $\varphi: \mathcal{H}_{n} \rightarrow \mathbb{R} \times \mathbb{R} \times \mathbb{C}^{m-1}$ is an isomorphism of real associative algebras. Our goal is to use Theorem 2.1 by assigning $\mathcal{B}=\mathbb{R} \times \mathbb{R} \times \mathbb{C}^{m-1}$ the appropriate weighted norm as to make (10) a dilation. After some experimentation, one finds that

$$
\left\|\left(a, b, z_{1}, z_{2}, \ldots, z_{m-1}\right)\right\|=\sqrt{a^{2}+b^{2}+2\left(\left|z_{1}\right|^{2}+\cdots+\left|z_{m-1}\right|^{2}\right)}
$$

serves our purpose. Considering $p(j)=\sum_{k=0}^{2 m-1} a_{k} j^{k}$,

$$
\begin{aligned}
\|\varphi(p(j))\|^{2}= & \left(\sum_{k=0}^{2 m-1} a_{k}\right)^{2}+\left(\sum_{k=0}^{2 m-1}(-1)^{k} a_{k}\right)^{2}+2 \sum_{s=1}^{m-1}\left|\sum_{k=0}^{2 m-1} a_{k} e^{\frac{i k s \pi}{m}}\right|^{2} \\
= & 2 \sum_{k=0}^{2 m-1} a_{k}^{2}+2 \sum_{p<q} a_{p} a_{q}\left(1+(-1)^{p+q}\right) \\
& +2 \sum_{s=1}^{m-1}\left[\left(\sum_{k=0}^{2 m-1} a_{k} \cos \left(\frac{k s \pi}{m}\right)\right)^{2}+\left(\sum_{k=0}^{2 m-1} a_{k} \sin \left(\frac{k s \pi}{m}\right)\right)^{2}\right] \\
= & 2 m \sum_{k=0}^{2 m-1} a_{k}^{2}+2 \sum_{p<q} a_{p} a_{q}\left(1+(-1)^{p+q}\right) \\
& +4 \sum_{s=1}^{m-1} \sum_{p<q} a_{p} a_{q}\left[\cos \left(\frac{p s \pi}{m}\right) \cos \left(\frac{q s \pi}{m}\right)+\sin \left(\frac{p s \pi}{m}\right) \sin \left(\frac{q s \pi}{m}\right)\right] \\
= & 2 m\|p(j)\|^{2}+2 \sum_{p<q} a_{p} a_{q}\left[1+(-1)^{p+q}+2 \sum_{s=1}^{m-1} \cos \left(\frac{(p-q) s \pi}{m}\right)\right] \\
= & 2 m\|p(j)\|^{2}+2 \sum_{p<q} a_{p} a_{q} \sum_{s=0}^{2 m-1} \cos \left(\frac{(p-q) s \pi}{m}\right) .
\end{aligned}
$$

Lemma 3.1 If $p, q, m$ are integers and $p<q$ then $\sum_{s=0}^{2 m-1} \cos \left(\frac{(p-q) s \pi}{m}\right)=0$.

Proof If $p-q$ is not a multiple of $m$ then let $\theta=\pi / m$ and note the points $1, e^{i \theta}, \ldots, e^{i(2 m-1) \theta}$ form a set of $2 m$-symmetrically arranged points on the unit circle whose centroid is the origin. Since $e^{i \theta}=\cos \theta+i \sin \theta$, we identify $\frac{1}{n} \sum_{s=0}^{2 m-1} \cos \left(\frac{(p-q) s \pi}{m}\right)$ as the real coordinate of the centroid. If $p-q$ is a multiple of $m$ then $\cos \left(\frac{(p-q) s \pi}{m}\right)=(-1)^{s}$ and the lemma follows. 
In view of Lemma 3.1 and (12), we find $\|\varphi(z)\|^{2}=2 m\|z\|^{2}$ for each $z \in \mathcal{H}_{n}$. Thus $\varphi$ is a dilation with constant $v=\sqrt{2 m}$. Let $\mathcal{B}=\mathbb{R}^{2} \times \mathbb{C}^{m-1}$ have norm as in (11). Then Theorem 2.2 indicates the submultiplicative constant of $\mathcal{B}$ is $m_{\mathcal{B}}=1$. Therefore, Theorem 2.1 provides $m_{\mathcal{H}_{n}}=v m_{\mathcal{B}}$, thus $m_{\mathcal{H}_{n}}=\sqrt{2 m}=\sqrt{n}$.

\section{2 $\mathcal{H}_{n}$ for odd $n$}

Notice that if $n=2 m+1$ then we have the following factorization of $x^{n}-1$ :

$$
x^{n}-1=(x-1) \prod_{k=1}^{m}\left(x-e^{\frac{2 \pi k i}{2 m+1}}\right)\left(x-e^{-\frac{2 \pi k i}{2 m+1}}\right) \text {. }
$$

As in (10) we define an isomorphism $\varphi: \mathcal{H}_{2 m+1} \rightarrow \mathbb{R} \times \mathbb{C}^{m}$ for each $p(j) \in \mathcal{H}_{2 m+1}$ by

$$
\varphi(p(j))=\left(p(1), p\left(e^{\frac{2 \pi i}{2 m+1}}\right), p\left(e^{\frac{4 \pi i}{2 m+1}}\right), \ldots, p\left(e^{\frac{2 m \pi i}{2 m+1}}\right)\right)
$$

Let $\mathcal{B}=\mathbb{R} \times \mathbb{C}^{m}$ have norm

$$
\left\|\left(a, z_{1}, \ldots, z_{m}\right)\right\|=\sqrt{a^{2}+2\left(\left|z_{1}\right|^{2}+\cdots+\left|z_{m}\right|^{2}\right)} .
$$

Thus for $p(j)=\sum_{k=0}^{2 m} a_{k} j^{k}$,

$$
\begin{aligned}
\|\varphi(p(j))\|^{2}= & \left(\sum_{k=0}^{2 m} a_{k}\right)^{2}+2 \sum_{s=1}^{m} \mid \sum_{k=0}^{2 m} a_{k} e^{\left.i \frac{i 2 k s \pi}{2 m+1}\right|^{2}} \\
= & \sum_{k=0}^{2 m} a_{k}^{2}+2 \sum_{p<q} a_{p} a_{q} \\
& +2 \sum_{s=1}^{m}\left[\left(\sum_{k=0}^{2 m} a_{k} \cos \left(\frac{2 k s \pi}{2 m+1}\right)\right)^{2}+\left(\sum_{k=0}^{2 m} a_{k} \sin \left(\frac{2 k s \pi}{2 m+1}\right)\right)^{2}\right] \\
= & (2 m+1) \sum_{k=0}^{2 m} a_{k}^{2}+2 \sum_{p<q} a_{p} a_{q} \\
& +4 \sum_{s=1}^{m} \sum_{p<q} a_{p} a_{q}\left[\cos \left(\frac{2 s p \pi}{2 m+1}\right) \cos \left(\frac{2 s q \pi}{2 m+1}\right)\right. \\
& \left.+\sin \left(\frac{2 s p \pi}{2 m+1}\right) \sin \left(\frac{2 s q \pi}{2 m+1}\right)\right] \\
= & (2 m+1)\|p(j)\|^{2}+2 \sum_{p<q} a_{p} a_{q}\left[1+2 \sum_{s=1}^{m} \cos \left(\frac{2 s(p-q) \pi}{2 m+1}\right)\right] \\
& \left(2 m p(j) \|^{2}+2 \sum_{p<q} a_{p} a_{q} \sum_{s=0} \cos \left(\frac{2 s(p-q) \pi}{2 m+1}\right)\right.
\end{aligned}
$$

The proof of Lemma 3.1 is easily adapted to show the following result:

Lemma 3.2 If $p, q, m$ are integers and $p<q$ then $\sum_{s=0}^{2 m} \cos \left(\frac{2 s(p-q) \pi}{2 m+1}\right)=0$. 
In view of the Lemma 3.2 and (16), we find $\|\varphi(z)\|^{2}=(2 m+1)\|z\|^{2}$ for each $z \in \mathcal{H}_{n}$ where $n=2 m+1$. Thus $\varphi$ is a dilation with constant $v=\sqrt{2 m+1}$. Let $\mathcal{B}=\mathbb{R} \times \mathbb{C}^{m}$ have norm as in (15). Then Theorem 2.2 indicates that the submultiplicative constant of $\mathcal{B}$ is $m_{\mathcal{B}}=1$. Therefore, Theorem 2.1 provides $m_{\mathcal{H}_{n}}=v m_{\mathcal{B}}$ and thus $m_{\mathcal{H}_{n}}=\sqrt{2 m+1}=\sqrt{n}$.

Theorem 3.3 If $\mathcal{H}_{n}$ is given norm $\left\|a_{0}+a_{1} j+\cdots+a_{n} j^{n-1}\right\|=\sqrt{a_{0}^{2}+a_{2}^{2}+\cdots+a_{n}^{2}}$ then $m_{\mathcal{H}_{n}}=$ $\sqrt{n}$. Moreover, for $r \geq 2$ we find $m_{r}\left(\mathcal{H}_{n}\right)=n^{(r-1) / 2}$ and $\rho_{\mathcal{H}_{n}}=\sqrt{n}$.

Proof We have already proved $m_{\mathcal{H}_{n}}=\sqrt{n}$. Consider $x=1+j+j^{2}+\cdots+j^{n-1}$ and observe $x^{2}=n x$, hence $x^{r}=n^{r-1} x$ for $r \in \mathbb{N}$. Moreover, $\|x\|=\sqrt{n}$, thus $\left\|x^{r}\right\|=m_{\mathcal{H}_{n}}^{r-1}\|x\|^{r}$ and Proposition 1.2 provides $m_{r}\left(\mathcal{H}_{n}\right)=n^{(r-1) / 2}$. Finally, calculate

$$
\rho_{\mathcal{H}_{n}}=\limsup _{r \rightarrow \infty} \sqrt[r]{m_{r}\left(\mathcal{H}_{n}\right)}=\limsup _{r \rightarrow \infty} \sqrt[r]{n^{(r-1) / 2}}=\sqrt{n}
$$

It is interesting to note that the inequality $\|x y\| \leq \sqrt{n}\|x\|\|y\|$ is sharp for $x=y=1+j+$ $j^{2}+\cdots+j^{n-1}$ since $\|x\|=\sqrt{n}$ and as

$$
x^{2}=\left(1+j+j^{2}+\cdots+j^{n-1}\right)^{2}=n\left(1+j+j^{2}+\cdots+j^{n-1}\right)=n x .
$$

Thus $\|x y\|=n\|x\|=n \sqrt{n}=\sqrt{n}\|x\|\|y\|$. The same $x$ has $x(x-n)=0$, which shows $x$ and $x-n$ are zero-divisors. This illustrates Remark 2.3.

\section{Submultiplicative constant of the complicated numbers}

We follow [3] and say $\mathcal{C}_{n}=\left\{a_{0}+a_{1} k+\cdots+a_{n-1} k^{n-1} \mid a_{i} \in \mathbb{R}, k^{n}=-1\right\}$ is the set of complicated numbers. Let us assume the norm on $\mathcal{C}_{n}$ is given by

$$
\left\|a_{0}+a_{1} k+\cdots+a_{n-1} k^{n-1}\right\|=\sqrt{a_{0}^{2}+\cdots+a_{n-1}^{2}}
$$

throughout this section.

Theorem 4.1 The submultiplicative constant

$$
m_{\mathcal{C}_{n}}= \begin{cases}\sqrt{n / 2} & \text { if } n \text { is even, } \\ \sqrt{n} & \text { ifn is odd }\end{cases}
$$

Proof If $n$ is odd then $\Psi(k)=-j$ defines an isomorphism of $\mathcal{C}_{n}$ and $\mathcal{H}_{n}$ since $\Psi(k)^{n}=(-j)^{n}=$ $(-1)^{n} j^{n}=-1$. Extending $\Psi$ to arbitrary elements,

$$
\Psi\left(a_{0}+a_{1} k+\cdots+a_{n-1} k^{n-1}\right)=a_{0}-a_{1} j+a_{2} j^{2}+\cdots+a_{n-1} j^{n-1} .
$$

If $\left\|a_{0}+a_{1} k+\cdots+a_{n-1} k^{n-1}\right\|=\sqrt{a_{0}^{2}+\cdots+a_{n-1}^{2}}$ then using the norm before discussed for $\mathcal{H}_{n}$ observe $\|\Psi(z)\|=\|z\|$. Therefore, from Theorem 2.1 we find $m_{\mathcal{C}_{n}}=m_{\mathcal{H}_{n}}$.

Consider $\mathcal{C}_{n}$ for $n=2 m$ with $m \geq 1$. Let $\theta_{j}=\frac{\pi}{n}(2 j+1)$ and $x_{j}=\exp \left(i \theta_{j}\right)$ for $j=0,1, \ldots$, $n / 2-1$. Define $\varphi: \mathcal{C}_{n} \rightarrow \mathbb{C}^{n / 2}$ by

$$
\varphi(f(k))=\left(f\left(x_{0}\right), \ldots, f\left(x_{n / 2-1}\right)\right)
$$


If $f(k)=a_{0}+a_{1} k+\cdots+a_{n-1} k^{n-1} \in \mathcal{C}_{n}$ then calculate

$$
\begin{aligned}
\left|f\left(x_{j}\right)\right|^{2} & =\left|\sum_{q=1}^{n-1} a_{q} \exp \left(i q \theta_{j}\right)\right|^{2} \\
& =\left|\sum_{q=1}^{n-1} a_{q}\left[\cos \left(q \theta_{j}\right)+i \sin \left(q \theta_{j}\right)\right]\right|^{2} \\
& =\left[\sum_{q=1}^{n-1} a_{q} \cos \left(q \theta_{j}\right)\right]^{2}+\left[\sum_{q=1}^{n-1} a_{q} \sin \left(q \theta_{j}\right)\right]^{2} \\
& =\sum_{q=1}^{n-1} a_{q}^{2}+\sum_{p<q} a_{p} a_{q}\left[\cos \left(p \theta_{j}\right) \cos \left(q \theta_{j}\right)+\sin \left(p \theta_{j}\right) \sin \left(q \theta_{j}\right)\right] \\
& =\|f(k)\|^{2}+\sum_{p<q} a_{p} a_{q} \cos \left((p-q) \theta_{j}\right) .
\end{aligned}
$$

Observe that, much for the same reasons as given for Lemma 3.1,

$$
\sum_{j=0}^{n / 2-1} \cos \left((p-q) \theta_{j}\right)=\sum_{j=0}^{n / 2-1} \cos \left(\frac{\pi(p-q)(2 j+1)}{2 m}\right)=0
$$

If $\mathbb{C}^{n / 2}$ has norm $\left\|\left(z_{1}, \ldots, z_{n / 2}\right)\right\|^{2}=\left|z_{1}\right|^{2}+\cdots+\left|z_{n / 2}\right|^{2}$ then Theorem 2.2 indicates $m_{\mathbb{C}^{n / 2}}=1$. Notice (21) and (22) yield

$$
\|\varphi(f(k))\|^{2}=\sum_{j=0}^{n / 2-1}\left|f\left(x_{j}\right)\right|^{2}=\frac{n}{2}\|f(k)\|^{2} .
$$

Consequently, $\varphi$ is a dilation with $v=\sqrt{n / 2}$, and Theorem 2.1 provides $m_{\mathcal{C}_{n}}=\sqrt{n / 2}$.

In the special case $n=2$, we note $\mathcal{C}_{2}$ has a multiplicative norm. However, for other $n>$ 2 the norm is not multiplicative. Furthermore, $\|x y\|=m_{\mathcal{C}_{n}}\|x\|\|y\|$ only for select $x, y$. In particular, if $n$ is odd then $x=1-k+k^{2}-\cdots+k^{n-1} \in \mathcal{C}_{n}$ has $x^{2}=n x$, thus $\left\|x^{2}\right\|=\sqrt{n}\|x\|^{2}$. In contrast, for even $n \neq 2$ notice $x=\sum_{j=0}^{n-1} \cos \left(\frac{j \pi}{n}\right) k^{j}$ is a nonzero zero-divisor with $x^{2}=\frac{n}{2} x$ and $\|x\|=\sqrt{\frac{n}{2}}$. Thus $\left\|x^{2}\right\|=\sqrt{\frac{n}{2}}\|x\|^{2}$. Once again, Remark 2.3 is illustrated.

\section{Submultiplicative constant of the dual numbers}

The $n$-dual number is defined as an element of $\Delta_{n}=\mathbb{R}[x] /\left\langle x^{n}\right\rangle$, or equivalently, $a_{0}+a_{1} \varepsilon+$ $\cdots+a_{n-1} \varepsilon^{n-1}$ where $\varepsilon^{n}=0$. We study the norm on $\Delta_{n}$ defined by

$$
\left\|a_{0}+a_{1} \varepsilon+\cdots+a_{n-1} \varepsilon^{n-1}\right\|=\sqrt{a_{0}^{2}+a_{1}^{2}+\cdots+a_{n-1}^{2}}
$$

Theorem 5.1 The submultiplicative constant for $\Delta_{n}$ is

(1) for $n=2, m_{2}\left(\Delta_{2}\right)=\frac{2}{\sqrt{3}}$;

(2) for $n=3, m_{2}\left(\Delta_{3}\right)=\frac{4}{3}$. 
Proof In all of the cases, we mainly use the AM-GM inequality. Begin with claim (1). Suppose $x=a_{0}+a_{1} \varepsilon, y=b_{0}+b_{1} \varepsilon$ and observe:

$$
\begin{aligned}
4\|x\|^{2} \cdot\|y\|^{2} & =4\left(a_{0}^{2}+a_{1}^{2}\right)\left(b_{0}^{2}+b_{1}^{2}\right) \\
& =3\left(a_{0}^{2} b_{0}^{2}+a_{1}^{2} b_{0}^{2}+a_{0}^{2} b_{1}^{2}\right)+\left(a_{0}^{2} b_{0}^{2}+4 a_{1}^{2} b_{1}^{2}\right)+\left(a_{1}^{2} b_{0}^{2}+a_{0}^{2} b_{1}^{2}\right) \\
& \geq 3\left(a_{0}^{2} b_{0}^{2}+a_{1}^{2} b_{0}^{2}+a_{0}^{2} b_{1}^{2}\right)+4 a_{0} b_{0} a_{1} b_{1}+2 a_{1} b_{0} a_{0} b_{1} \\
& =3\left[\left(a_{0} b_{0}\right)^{2}+\left(a_{1} b_{0}+a_{0} b_{1}\right)^{2}\right]=3\left\|a_{0} b_{0}+\left(a_{1} b_{0}+a_{0} b_{1}\right) \varepsilon\right\|^{2} \\
& =3\|x y\|^{2} .
\end{aligned}
$$

If we set $a_{0}=b_{0}=\sqrt{2}$ and $a_{1}=b_{1}=1$ then equality is attained. Next, prove claim (2). We suppose $x=a_{0}+a_{1} \varepsilon+a_{2} \varepsilon^{2}$ and $y=b_{0}+b_{1} \varepsilon+b_{2} \varepsilon^{2}$. Calculate:

$$
\begin{aligned}
16\|x\|^{2} \cdot\|y\|^{2}= & 16\left(a_{0}^{2}+a_{1}^{2}+a_{2}^{2}\right)\left(b_{0}^{2}+b_{1}^{2}+b_{2}^{2}\right) \\
= & 9\left(a_{0}^{2} b_{0}^{2}+a_{1}^{2} b_{0}^{2}+a_{0}^{2} b_{1}^{2}+a_{0}^{2} b_{2}^{2}+a_{1}^{2} b_{1}^{2}+a_{2}^{2} b_{0}^{2}\right)+16 a_{1}^{2} b_{2}^{2}+4 a_{0}^{2} b_{1}^{2} \\
& +16 a_{2}^{2} b_{1}^{2}+4 a_{1}^{2} b_{0}^{2}+16 a_{2}^{2} b_{2}^{2}+a_{0}^{2} b_{0}^{2}+3\left(a_{0}^{2} b_{1}^{2}+a_{1}^{2} b_{0}^{2}\right) \\
& +6\left(a_{0}^{2} b_{0}^{2}+a_{1}^{2} b_{1}^{2}\right)+\frac{1}{2} a_{1}^{2} b_{1}^{2}+2 a_{2}^{2} b_{0}^{2}+\frac{1}{2} a_{1}^{2} b_{1}^{2}+2 a_{0}^{2} b_{2}^{2}+5\left(a_{0}^{2} b_{2}^{2}+a_{2}^{2} b_{0}^{2}\right) \\
\geq & 9\left(a_{0}^{2} b_{0}^{2}+a_{1}^{2} b_{0}^{2}+a_{0}^{2} b_{1}^{2}+a_{0}^{2} b_{2}^{2}+a_{1}^{2} b_{1}^{2}+a_{2}^{2} b_{0}^{2}\right) \\
& +16 a_{1} b_{2} a_{0} b_{1}+16 a_{2} b_{1} a_{1} b_{0}+8 a_{2} b_{2} a_{0} b_{0}+6 a_{0} b_{1} a_{1} b_{0} \\
& +12 a_{0} b_{0} a_{1} b_{1}+2 a_{1} b_{1} a_{2} b_{0}+2 a_{1} b_{1} a_{0} b_{2}+10 a_{0} b_{2} a_{2} b_{0} \\
= & 9\left[\left(a_{0} b_{0}\right)^{2}+\left(a_{1} b_{0}+a_{0} b_{1}\right)^{2}+\left(a_{0} b_{2}+a_{1} b_{1}+a_{2} b_{0}\right)^{2}\right] \\
= & 9\left\|a_{0} b_{0}+\left(a_{1} b_{0}+a_{0} b_{1}\right) \varepsilon+\left(a_{0} b_{2}+a_{1} b_{1}+a_{2} b_{0}\right) \varepsilon^{2}\right\|^{2} \\
= & 9\|x y\|^{2} .
\end{aligned}
$$

Equality occurs if, for instance, $a_{0}=b_{0}=a_{1}=b_{1}=2, a_{2}=b_{2}=1$.

It is interesting to note that in both cases $(n=2,3)$ we find equality occurs when

$$
\frac{a_{0}}{b_{0}}=\frac{a_{1}}{b_{1}}=\frac{a_{2}}{b_{2}} .
$$

Assuming the above trend continues, we conjecture that:

(1) for $n=4, m_{2}\left(\Delta_{4}\right)=\sqrt{\frac{2(1103+33 \sqrt{33})}{1153}} \approx 1.49736$. We note equality in $\|x y\| \leq m_{2}\left(\Delta_{4}\right)\|x\|\|y\|$ is attained for $x=y=$ $\sqrt{5+\sqrt{\frac{11}{3}}}+\left(1+\sqrt{\frac{11}{3}}\right) \varepsilon+\sqrt{3+\sqrt{\frac{11}{3}}} \varepsilon^{2}+\varepsilon^{3} \approx 2.62961+2.91485 \varepsilon+2.21695 \varepsilon^{2}+\varepsilon^{3}$.

(2) for $n=5$, the closed form expression is too lengthy for us to display here, however, an approximation of the constant is $m_{2}\left(\Delta_{5}\right) \approx 1.64748$. Equality in $\|x y\| \leq m_{2}\left(\Delta_{5}\right)\|x\|\|y\|$ is attained when $x=y \approx 1-1.14862 \varepsilon+1.03046 \varepsilon^{2}-0.700308 \varepsilon^{3}+0.304849 \varepsilon^{4}$.

(3) for $n=6$, the submultiplicative constant is $m_{2}\left(\Delta_{6}\right) \approx 1.78611$ and equality in $\|x y\| \leq m_{2}\left(\Delta_{6}\right)\|x\|\|y\|$ is attained at $x=y \approx 1-1.16152 \varepsilon+1.12963 \varepsilon^{2}-0.915093 \varepsilon^{3}+0.589126 \varepsilon^{4}-0.253601 \varepsilon^{5}$. 
Theorem 5.2 For $r \in \mathbb{N}, r \geq 2$, we have $m_{r}\left(\Delta_{2}\right)=\sqrt{\frac{r+1}{\left(1+\frac{1}{r}\right)^{r}}}$.

Proof Considering $a_{i}, b_{i} \in \mathbb{R}$ for $1 \leq i \leq r$, since $\varepsilon^{2}=0$, we find

$$
\prod_{i=1}^{r}\left(a_{i}+b_{i} \varepsilon\right)=\prod_{i=1}^{r} a_{i}+\varepsilon \sum_{i=1}^{r}\left(\prod_{j \neq i} a_{j}\right) b_{i}
$$

Let $g^{2}$ be the smallest constant such that the inequality below holds for arbitrary $a_{i}, b_{i}$,

$$
g^{2} \prod_{i=1}^{r}\left(a_{i}^{2}+b_{i}^{2}\right) \geq \prod_{i=1}^{r} a_{i}^{2}+\left[\sum_{i=1}^{r}\left(\prod_{j \neq i} a_{j}\right) b_{i}\right]^{2}
$$

Substituting $a_{i}=1$ and $b_{i}=0$, we have $g^{2} \geq 1$. If there is at least one $a_{i}=0$ then, without loss of generality, we may suppose $a_{r}=0$, and thus for all $a_{i}, b_{i} \in \mathbb{R}$

$$
g^{2} \prod_{i=1}^{r-1}\left(a_{i}^{2}+b_{i}^{2}\right) b_{r}^{2} \geq \prod_{i=1}^{r-1}\left(a_{i}^{2}+b_{i}^{2}\right) b_{r}^{2} \geq a_{1}^{2} a_{2}^{2} \cdots a_{r-1}^{2} b_{r}^{2} .
$$

Thus (29) holds trivially. Therefore, let us suppose all $a_{i}$ are non-zero. Then we can normalize the above inequality to (setting $x_{i}=b_{i} / a_{i}$ )

$$
g^{2} \prod_{i=1}^{r}\left(1+x_{i}^{2}\right) \geq 1+\left(\sum_{i=1}^{r} x_{i}\right)^{2}
$$

Define $f\left(x_{1}, \ldots, x_{r}\right)=\frac{1+\left(x_{1}+\cdots+x_{r}\right)^{2}}{\left(1+x_{1}^{2}\right) \cdots\left(1+x_{r}^{2}\right)}$ and note by construction $g^{2} \geq f\left(x_{1}, \ldots, x_{r}\right)$ for all $x_{1}, \ldots, x_{r}$. We seek solutions to $\nabla f=0$ in the interest of finding the global maximum of $f$. Set $S=x_{1}+x_{2}+\cdots+x_{n}$ and calculate

$$
\frac{\partial f}{\partial x_{i}}=-2 \frac{\left(S-x_{i}\right)\left(x_{i} S-1\right)}{\left(1+x_{i}^{2}\right) \prod_{k=1}^{r}\left(1+x_{k}^{2}\right)} .
$$

Hence, $\nabla f=0$ if and only if $\left(S-x_{i}\right)\left(x_{i} S-1\right)=0$ for all $1 \leq i \leq r$.

(1) If $S=0$ then $\left(S-x_{i}\right)\left(x_{i} S-1\right)=0$ implies $x_{1}=0, x_{2}=0, \ldots, x_{r}=0$.

(2) Suppose $x_{1}, x_{2}, \ldots, x_{r} \neq 0$. Then $\left(S-x_{i}\right)\left(x_{i} S-1\right)=0$ yields

$$
S+\frac{1}{S}=x_{1}+\frac{1}{x_{1}}=x_{2}+\frac{1}{x_{2}}=\cdots=x_{r}+\frac{1}{x_{r}},
$$

and we find solution $x_{1}=x_{2}=\cdots=x_{r}= \pm \frac{1}{\sqrt{r}}$.

Observe $f(0, \ldots, 0)=1$ whereas $f\left(\frac{1}{\sqrt{r}}, \ldots, \frac{1}{\sqrt{r}}\right)=f\left(-\frac{1}{\sqrt{r}}, \ldots,-\frac{1}{\sqrt{r}}\right)=\frac{r+1}{\left(1+\frac{1}{r}\right)^{r}} \geq 1$ for all $r \geq 2$. Therefore we conclude $m_{r}\left(\Delta_{2}\right)=\sqrt{g}=\sqrt{\frac{r+1}{\left(1+\frac{1}{r}\right)^{r}}}$.

Let $\Delta_{n}$ have $p$-norm defined for $p<\infty$ by

$$
\left\|a_{1}+a_{2} \varepsilon+\cdots+a_{n} \varepsilon^{n-1}\right\|=\sqrt[p]{a_{1}^{p}+a_{2}^{p}+\cdots+a_{n}^{p}} .
$$

Theorem 5.3 Suppose $p \in(1, \infty)$. If $\Delta_{n}$ is endowed with $p$-norm then $\rho_{\Delta_{n}}=1$. 
Proof Let it be understood that $m_{r}\left(\Delta_{n}\right)$ is defined with respect to the $p$-norm throughout this proof. Suppose $a_{i, j}>0$. We will attempt to bound $m_{r}\left(\Delta_{n}\right)$ by an appropriate constant. We have

$$
\prod_{i=1}^{r} \sum_{j=0}^{n-1} a_{i, j} \varepsilon^{j}=\sum_{k=0}^{n-1}\left(\sum_{d_{1}+\cdots+d_{r}=k} \prod_{i=1}^{r} a_{i, d_{i}}\right) \varepsilon^{k} .
$$

Therefore,

$$
m_{r}\left(\Delta_{n}\right)^{p} \prod_{i=1}^{r} \sum_{j=0}^{n-1} a_{i, j}^{p} \geq \sum_{k=0}^{n-1}\left(\sum_{d_{1}+\cdots+d_{r}=k} \prod_{i=1}^{r} a_{i, d_{i}}\right)^{p} .
$$

A few observations about (35) are as follows:

(1) There are $\left(\begin{array}{c}k+r-1 \\ k\end{array}\right)$ distinct terms $\prod_{i=1}^{r} a_{i, d_{i}}$ in the sum

$$
\sum_{d_{1}+\cdots+d_{r}=k} \prod_{i=1}^{r} a_{i, d_{i}}
$$

(2) Each term $\prod_{i=1}^{r} a_{i, d_{i}}$ to the power $p$ occurs in the product

$$
\prod_{i=1}^{r} \sum_{j=0}^{n-1} a_{i, j}^{p}
$$

(3) $\left(\begin{array}{c}(k+1)+r-1 \\ k+1\end{array}\right)=\left(\begin{array}{c}k+r-1 \\ k\end{array}\right)+\left(\begin{array}{c}k+r-1 \\ k+1\end{array}\right) \geq\left(\begin{array}{c}k+r-1 \\ k\end{array}\right)$, so the sequence $\left(\begin{array}{c}k+r-1 \\ k\end{array}\right)$ is increasing in variable $k$ and thus $\max _{0 \leq k<n}\left\{\left(\begin{array}{c}k+r-1 \\ k\end{array}\right)\right\}=\left(\begin{array}{c}n+r-2 \\ n-1\end{array}\right)=O\left(r^{n-1}\right)$.

(4) By Hölder's inequality with $q=\frac{p}{p-1}$,

$$
x_{1} \cdot 1+x_{2} \cdot 1+\cdots+x_{s} \cdot 1 \leq\left(x_{1}^{p}+x_{2}^{p}+\cdots+x_{s}^{p}\right)^{\frac{1}{p}}\left(1^{q}+1^{q}+\cdots+1^{q}\right)^{\frac{1}{q}} .
$$

Consequently, $\left(x_{1}+x_{2}+\cdots+x_{s}\right)^{p} \leq s^{p-1}\left(x_{1}^{p}+x_{2}^{p}+\cdots+x_{s}^{p}\right)$.

Combining the observations above, we find:

$$
\begin{aligned}
\sum_{k=0}^{n-1}\left(\sum_{d_{1}+\cdots+d_{r}=k} \prod_{i=1}^{r} a_{i, d_{i}}\right)^{p} & \leq \sum_{k=0}^{n-1}\left(\begin{array}{c}
k+r-1 \\
k
\end{array}\right)^{p-1}\left(\sum_{d_{1}+\cdots+d_{r}=k} \prod_{i=1}^{r} a_{i, d_{i}}^{p}\right) \\
& \leq\left(\begin{array}{c}
n+r-2 \\
n-1
\end{array}\right)^{p-1} \sum_{k=0}^{n-1}\left(\sum_{d_{1}+\cdots+d_{r}=k} \prod_{i=1}^{r} a_{i, d_{i}}^{p}\right) \\
& \leq\left(\begin{array}{c}
n+r-2 \\
n-1
\end{array}\right)^{p} \prod_{i=1}^{r} \sum_{j=0}^{n-1} a_{i, j}^{p} .
\end{aligned}
$$

Thus,

$$
m_{r}\left(\Delta_{n}\right) \leq\left(\begin{array}{c}
n+r-2 \\
n-1
\end{array}\right)=O\left(r^{n-1}\right)
$$

Since $n$ is fixed, $\rho_{\Delta_{n}} \leq \limsup _{r \rightarrow \infty} \sqrt[r]{r^{n-1}}=1$. However, we found in (3) that $\rho_{\Delta_{n}} \geq$ $\|1\|^{-1}=1$. Thus, $\rho_{\Delta_{n}}=\|1\|^{-1}=1$. 


\section{Submultiplicative constants for the dualplex numbers}

An $n$-dualplex number is defined as an element of $\mathcal{A}=\mathbb{R}[x] /\left\langle\left(x^{2}+1\right)^{n}\right\rangle$, or equivalently, $a_{0}+a_{1} q+\cdots+a_{2 n-1} q^{2 n-1}$ where $a_{i} \in \mathbb{R}$ and $\left(q^{2}+1\right)^{n}=0$. If we use norm

$$
\left\|a_{0}+a_{1} q+\cdots+a_{2 n-1} q^{2 n-1}\right\|=\sqrt{a_{0}^{2}+a_{1}^{2}+\cdots+a_{2 n-1}^{2}}
$$

then for $n=1$ we have complex numbers with the usual multiplicative norm and multiplicative constant 1 . However, for $n=2$ we conjecture that the submultiplicative constant is given by $m_{\mathcal{A}}=\sqrt{\frac{71+17 \sqrt{17}}{6}}$ where $\|x y\|=m_{\mathcal{A}}\|x\|\|y\|$ if we set $x=y=(3-\sqrt{17}) q+2 q^{3}$.

\section{Convergence of geometric series over some algebras}

In this section we use Theorem 1.3 in conjunction with the results of the previous sections to study the geometric series over an algebra.

Example 7.1 Consider the geometric series $\sum_{n=0}^{\infty} z^{n}$ in the $N$-hyperbolic numbers $\mathcal{H}_{N}$. Observe $c_{n}=1$ and $C=\limsup _{n \rightarrow \infty} \sqrt[n]{\left\|c_{n}\right\|}=1$. We found $\rho_{\mathcal{H}_{N}}=\sqrt{N}$ in Theorem 3.3. Theorem 1.3 gives that the geometric series converges for $\|z\|<\frac{1}{\sqrt{N}}$. Suppose $R>\frac{1}{\sqrt{N}}$, pick $z=\frac{R}{\sqrt{N}}\left(1+j+j^{2}+\cdots+j^{N-1}\right)$, and notice $\|z\|=R$ and that the geometric series diverges at $z$ by the Root Test as

$$
\begin{aligned}
\limsup _{n \rightarrow \infty} \sqrt[n]{\left\|z^{n}\right\|} & =\limsup _{n \rightarrow \infty} \sqrt[n]{\frac{R^{n}}{\sqrt{N}^{n}}\left\|N^{n-1}\left(1+j+j^{2}+\cdots+j^{N-1}\right)\right\|} \\
& =\limsup _{n \rightarrow \infty} \sqrt[n]{R^{n} \sqrt{N}^{n-1}}=R \sqrt{N}>1 .
\end{aligned}
$$

We see the radius of convergence $1 / \sqrt{N}$ is maximal.

Example 7.2 Consider the Dual Numbers $\Delta_{N}$ for some $N \geq 2$. Apply Theorems 5.3 and 1.3 to see why the geometric series $\sum_{n=0}^{\infty} z^{n}$ converges for $\|z\|<1$. Also, if $R>1$ then setting $z=R$ gives a point with $\|z\|>1$ where the geometric series diverges.

\section{Acknowledgements}

We thank Liberty University for supporting Math 495 in the Spring 2018 Semester

Funding

Not applicable.

Availability of data and materials

Not applicable.

Competing interests

The authors declare that they have no competing interests.

Authors' contributions

The authors read and approved the final manuscript.

\section{Publisher's Note}

Springer Nature remains neutral with regard to jurisdictional claims in published maps and institutional affiliations.

Received: 9 November 2018 Accepted: 17 January 2019 Published online: 28 January 2019

References

1. Cook, J.S.: Introduction to $\mathcal{A}$-Calculus. https://arxiv.org/abs/1708.04135

2. Cook, J.S., Freese, D.: Analysis of power series in the $\mathcal{A}$-calculus. Int. Math. Forum 13(10), 473-483 (2018)

3. Good, I.J.: Skew circulants and the theory of numbers. Fibonacci Q. 24(1), 47-60 (1986) 